\title{
COMPORTAMENTO GEOQUÍMICO DE METAIS EM SEDIMENTOS DE MANGUEZAL DA BAÍA DE CAMAMU-BAHIA
}

\author{
OLIVEIRA, O.M.C. ${ }^{1}$; CRUZ, M.J.M. ${ }^{2} \&$ QUEIROZ, A.F.S. ${ }^{3}$ \\ ${ }_{1}$ Departamento de Geologia e Geofísica Aplicada (DGGA) e Núcleo de Estudos Ambientais (N.E.A.) - \\ Instituto de Geociências, Universidade Federal da Bahia, Rua Barão de Geremoabo, s/n, Campus de \\ Ondina, Sala 314-A, CEP 40170-290, Salvador - Bahia - olivia@ufba.br \\ ${ }^{2}$ Departamento de Geoquímica (DGq), Instituto de Geociências, Universidade Federal da Bahia, Rua \\ Barão de Geremoabo, s/n, Campus de Ondina, Sala 314-A, CEP 40170-290, Salvador - Bahia - \\ jeronimo@ufba.br
}

\author{
${ }^{3}$ Departamento de Geoquímica (DGq) e Núcleo de Estudos Ambientais (N.E.A.) - Instituto de \\ Geociências, Universidade Federal da Bahia, Rua Barão de Geremoabo, s/n, Campus de Ondina, \\ Sala 314-A, CEP 40170-290, Salvador - Bahia - queiroz@ufba.br
}

\begin{abstract}
Oliveira, O.M.C.; Cruz, M.J.M. \& Queiroz, A.F.S. 2009. Metal's geochemistry behavior in mangrove sediments of the Camamu Bay-Bahia.Braz. J. Aquat. Sci. Technol. 13(2):1-8. ISSN 1808-7035. This inquiry encloses the sediments and mangroves of the Camamu Bay, Bahia, Brazil. 13 stations of sampling had been selected in which sediments, water and leaves were collected. Of these stations, 5 are located in mining zones and 8 , in the estuary of the rivers that flow down the bay. The samples had been separate by grain size, mineralogical characteristics, metals $(\mathrm{Pb}$, $\mathrm{Zn}, \mathrm{Cr}, \mathrm{Cu}, \mathrm{Cd}, \mathrm{Ba}, \mathrm{Mn}, \mathrm{Fe}$ and $\mathrm{Al}$ ), organic substances and nutrients. The results of the metals have evidenced differentiated geochemical behaviors for each one of the mangrove zones (compartment of the islands and around the Camamu Bay). The amounts of $\mathrm{Pb}, \mathrm{Zn}$ and $\mathrm{Cu}$, had been raised in the islands compartment, while $\mathrm{Mn}, \mathrm{Cr}, \mathrm{Al}$ and $\mathrm{Fe}$, presented higher concentration around the Bay. In relation to the dynamics of metal partition in the zones of mining of the interior islands, it was observed a preoccupying increase of $\mathrm{Pb}, \mathrm{Zn}, \mathrm{Mn}$ and $\mathrm{Ba}$.
\end{abstract}

Keywords: Mangrove, Sediments, Metals Extraction.

\section{INTRODUÇÃO}

A Baía de Camamu está localizada a cerca de $335 \mathrm{Km}$ a sul da cidade de Salvador, capital do Estado da Bahia (Figura 1). Esse acidente geográfico, de formato aproximadamente circular, é a terceira maior baía do Brasil. O seu interior é apinhado de numerosas ilhas, com destaque especial àquelas localizadas na porção central da baía, denominadas de ilhas Grande e Pequena.

Na Baía de Camamu os manguezais ocupam mais de $40 \mathrm{Km}^{2}$, constituindo-se um complexo natural de grande importância econômico de produção primária, com fornecimento de pescado para comercialização e consumo próprio das comunidades ribeirinhas.

Nas ilhas Grande e Pequena no interior da Baía de Camamu existem importantes mineralizações de barita - sulfato de bário $\left(\mathrm{BaSO}_{4}\right)$, que desde a década de 70 , tem sido processada a sua exploração industrial. Estas atividades, de origem antrópica, vêm influenciando o ecossistema local.

Assim, partículas provenientes de empilhamentos de minério são transportadas por ação eólica ou pela lixiviação de bacias de rejeito e os materiais têm sido carreados para o interior dos manguezais. Os elementos pesados associados a estes particulados são conjuntamente transportados e se constituem em um importante fator impactante ao ambiente local.

É mister assinalar que independente da origem do metal nos sedimentos, o seu tempo de residência depende de alguns processos que promovem sua fixação ou remobilização (Förstner \& Wittmann, 1981). Os processos de adsorção, co-precipitação com óxidos e hidróxidos de $\mathrm{Fe}$ e $\mathrm{Mn}$, complexação por moléculas orgânicas, além de mudanças de $\mathrm{pH}, \mathrm{E}_{\mathrm{H}}$, salinidade, podem promover a liberação parcial ou total das frações metálicas móveis. Por outro lado, a fração metálica incorporada na estrutura cristalina dos minerais não é solubilizável pelos processos físico-químicos comuns.

Este trabalho apresenta os resultados dos estudos geoquímicos e as conclusões obtidas sobre a dinâmica atuante provenientes das atividades exploratórias mineiras nos sítios da Baía de Camamu e suas ilhas interiores. 


\section{METODOLOGIA}

Para se atingir os objetivos propostos neste trabalho foram estabelecidas 13 (treze) estações de amostragem, distribuídas no interior da Baía de Camamu (Figura 1). Cinco destas estações foram localizadas nas ilhas centrais da baía (três na llha Grande e duas na llha Pequena) - Estações 1 a 5 - e oito na região do entorno da baía, em locais de confluência dos principais rios que desembocam na região estuarina lagunar, com o Oceano Atlântico Estações 6 a 13. Todas as estações foram posicionadas em zonas de abrangência do ecossistema de manguezal local.

Em cada estação foi amostrado substrato lamoso em áreas de manguezal, com testemunhos até $1 \mathrm{~m}$ de profundidade, seccionados em fatias que variaram de $20 \mathrm{~cm}$ em $20 \mathrm{~cm}$, do topo para a base a fim de permitir uma avaliação das possíveis variações das concentrações encontradas nas diversas seções seccionadas. Foram realizadas análises dos metais $\mathrm{Pb}$, $\mathrm{Zn}, \mathrm{Cr}, \mathrm{Cu}, \mathrm{Cd}, \mathrm{Ba}, \mathrm{Mn}, \mathrm{Fe}$ e Al em cada seção amostrada e em cada fatia seccionada, utilizando-se diferentes técnicas de extração (parcial, seqüencial e total), na fração do sedimento $<0,063 \mathrm{~mm}$. As análises foram realizadas nos Laboratórios de Geoquímica do Instituto de Geociências da Universidade Federal da Bahia.

Foram seguidas as seguintes metodologias:

Extração Parcial - Para este tipo de extração as amostras foram secas a $110^{\circ} \mathrm{C}$ por $2 \mathrm{~h}$, levadas a placa aquecedora após adição de $\mathrm{HNO}_{3}$ concentrado, segundo metodologia adotada por Linhares et al. (1995). Os metais $\mathrm{Pb}, \mathrm{Zn}, \mathrm{Cr}, \mathrm{Cu}, \mathrm{Cd}, \mathrm{Mn}, \mathrm{Fe}$ e Al foram determinados por Espectrofotometria de Absorção Atômica, com chama, marca Perkin Elmer, modelo 403, no Laboratório de Absorção Atômica do Departamento de Geoquímica do Instituto de Geociências da UFBA.
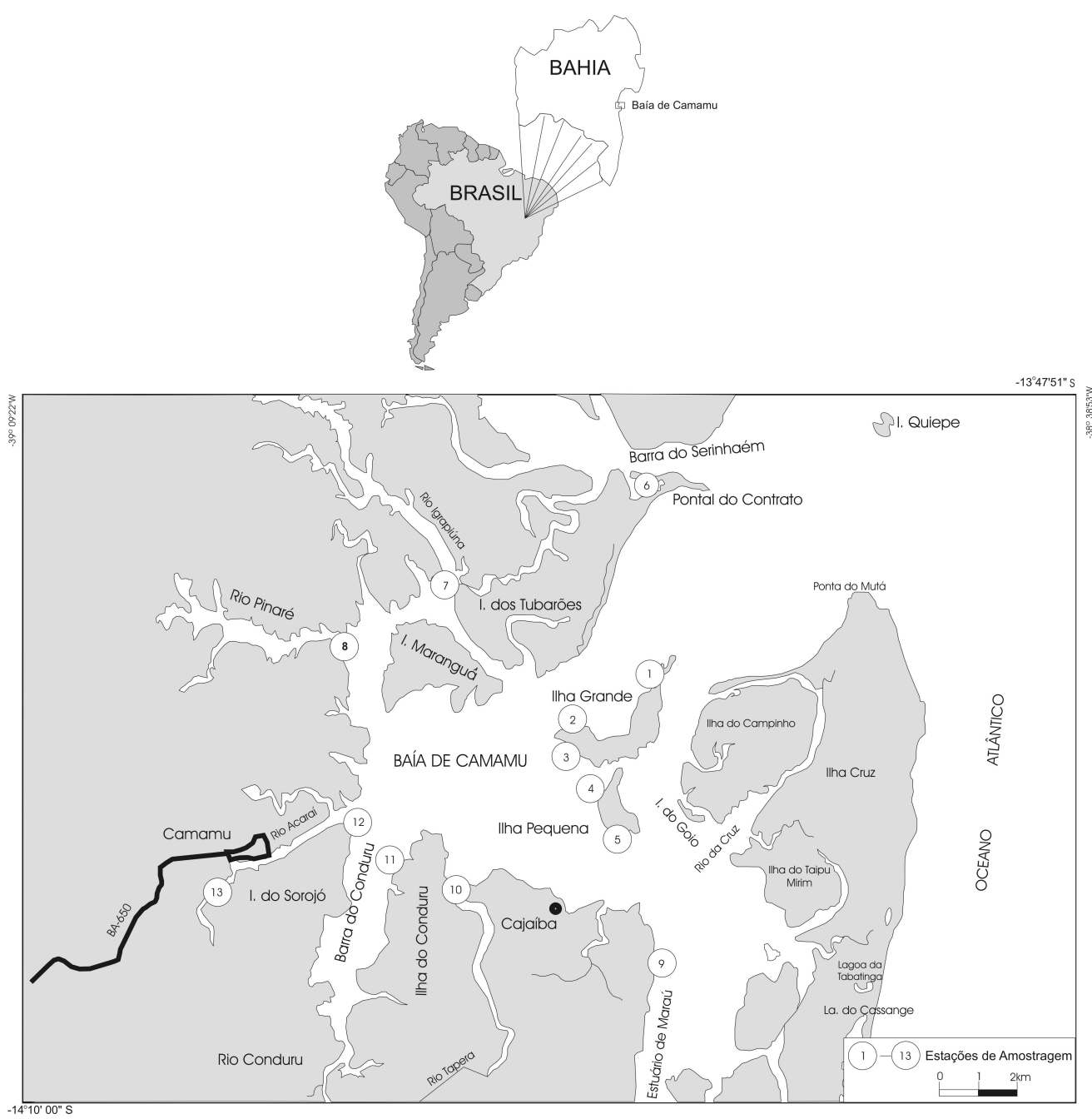

Figura 1 - Mapa de localização e amostragem da Baía de Camamu. 
Extração Seqüencial - A sorção de metais pesados em sedimentos pode ser avaliada investigando sua participação entre as várias fases geoquímicas. $\mathrm{O}$ procedimento de extração seqüencial que tem sido muito aplicado e discutido por diversos autores (Tessier et al., 1979; Fiszman et al., 1984; Lim \& Kiu, 1995, dentre outros) pode ser empregado para tal avaliação. Este procedimento é baseado no princípio de que metais sorvidos podem ser substituídos ou removidos de uma fase geoquímica pelo uso de extratores apropriados.

Cabral \& Lefebvre (1998), Bradl (2004) e Yong et al. (1993), têm questionado a perfeita seletividade dos reagentes utilizados, sobre a destruição apenas das ligações entre os contaminantes e a fase geoquímica selecionada. Dessa forma, os resultados da extração seqüencial podem ser interpretados como uma avaliação semi-quantitativa da partição dos contaminantes entre as fases geoquímicas ou como um bom indicador qualitativo.

O procedimento de extração seqüencial utilizado (Oliveira et al. 1998; Oliveira 2000) é ilustrado na Figura 2. A operacionalidade definida para a fração associada com cada fase e os reagentes usados em cada estágio é apresentada a seguir: Fase trocável -A fase metálica é liberada da superfície dos sólidos, tais como minerais de argila e óxidos bem cristalizados, através de atração ou repulsão eletrostática (Coulomb) (Greenland \& Hayes, 1978 in Cabral \& Lefebvre, 1998); Fase carbonática - $O$ alto conteúdo de carbonato aumenta o efeito tampão no sedimento e como conseqüência, aumenta a capacidade de retenção dos metais pesados. $\mathrm{O}$ acetato de sódio utilizado nessa fase quebra as ligações entre as espécies metálicas e o carbonato; Fase redutível - Capaz de quebrar as ligações entre os metais e os óxidos e hidróxidos de Fe e Mn, sem atacar os minerais silicáticos e as ligações entre os metais pesados e a matéria orgânica; Fase oxidável - Inclui os metais ligados à matéria orgânica e aos sulfetos oxidáveis, pois em condições oxidantes ocorre a degradação da matéria orgânica e os metais são removidos para a solução; Fração residual-É composta por metais presentes na estrutura cristalina dos minerais silicáticos. Somente através de digestão a quente é que estes metais são liberados.

Discussões complementares sobre as interações entre cada fase geoquímica e os metais, além da maneira como essas interações influenciam na efetividade dessas técnicas de extrações, foram realizadas por Cabral \& Lefebvre (1998), Fiszman et al. (1984) e Yong et al. (1992,1993).

As extrações foram realizadas em tubos de centrífuga de polietileno de $50 \mathrm{~mL}$ e cada fração foi separada por centrifugação a uma velocidade de 3000 rpm por 30 minutos. Foram determinados os metais $\mathrm{Pb}, \mathrm{Zn}, \mathrm{Cr}, \mathrm{Cu}, \mathrm{Cd}, \mathrm{Ba}, \mathrm{Mn}$, Fe e Al por ICP OES, seqüencial, marca Varian, modelo Liberty 150.

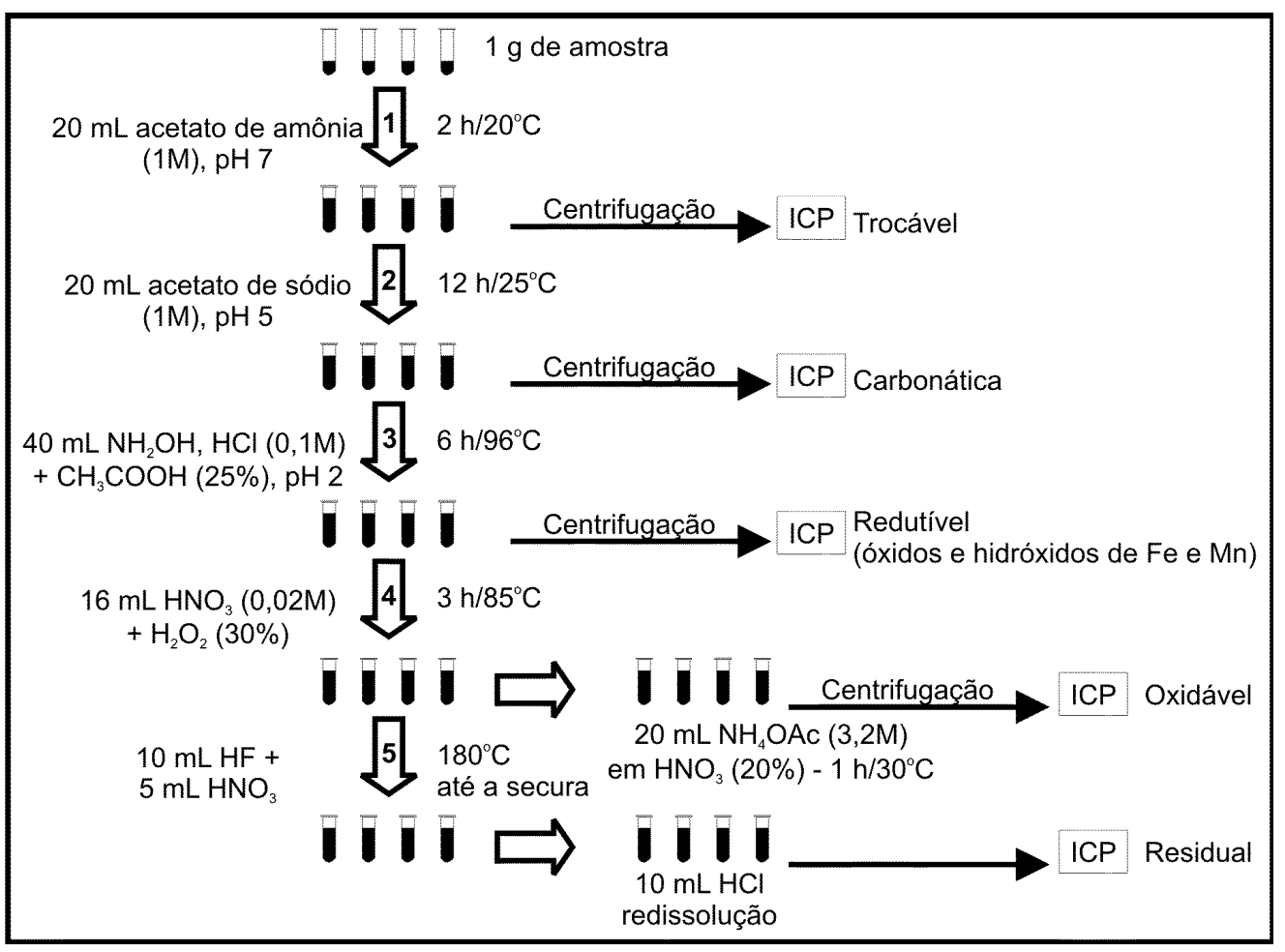

Figura 2 - Esquema de extração seqüencial utilizado para os sedimentos. 
A extração do elemento Ba na fração residual ficou impossibilitada pelo fato do ataque com $\mathrm{HF}+$ $\mathrm{HNO}_{3}$, utilizado para esse tipo de análise (Dolezal et al., 1968), não ter se mostrado eficiente para solubilizar o sulfato de bário totalmente.

A efetividade do processo de extração seqüencial pode ser julgado pela comparação da soma de cada fração com a quantidade de todos os metais extraídos (extração total). O erro admitido para esta comparação de aberturas é geralmente por volta de 10\% (Lee et al., 1997).

A decomposição ácida total foi realizada utilizando-se $\mathrm{HF}, \mathrm{HClO}_{4}$ e $\mathrm{HNO}_{3}$ na proporção 2:2:1. Os metais $\mathrm{Pb}, \mathrm{Zn}, \mathrm{Cr}, \mathrm{Cu}, \mathrm{Cd}, \mathrm{Mn}$, Fe e Al foram analisados por ICP/AES.

\section{RESULTADOS E DISCUSSÃO}

A distribuição dos metais nos sedimentos de manguezal da Baía de Camamu é caracterizada por diferentes ordens de proporção em cada fase analisada (Oliveira et al., 1998b), Figura 3. As concentrações apresentadas se referem a média daquelas encontradas em cada seção de $20 \mathrm{~cm}$ do perfil de $1 \mathrm{~m}$. A fração trocável apresenta os maiores percentuais relativos para o Mn em ambos os compartimentos, sendo seguido pelo $\mathrm{Pb}, \mathrm{Ba}$ e $\mathrm{Zn}$, no compartimento das ilhas, e pelo $\mathrm{Zn}, \mathrm{Ba}$ e $\mathrm{Pb}$, na região do entorno. A fração carbonática é representada preferencialmente pelo $\mathrm{Mn}$ em ambos os compartimentos, sendo seguido pelo $\mathrm{Ba}, \mathrm{Pb}$ e $\mathrm{Zn}$, na região das ilhas e pelo $\mathrm{Zn}, \mathrm{Ba}$ e $\mathrm{Pb}$, na região do entorno. A fração oxidável é representada preferencialmente pelo $\mathrm{Pb}, \mathrm{Mn}, \mathrm{Ba}$ e $\mathrm{Zn}$ nas ilhas e pelo $\mathrm{Mn}, \mathrm{Cr}, \mathrm{Zn}$ e $\mathrm{Pb}$, na região do entorno. A fração redutível é associada preferencialmente ao $\mathrm{Mn}$, em ambos os compartimentos, seguido pelo $\mathrm{Pb}, \mathrm{Ba}$ e $\mathrm{Zn}$, na região das ilhas e pelo $\mathrm{Zn}, \mathrm{Ba}$ e $\mathrm{Pb}$, na região do entorno. A fração residual é representada predominantemente pelo $\mathrm{Pb}, \mathrm{Mn}$ e $\mathrm{Zn}$ no compartimento das ilhas e pelo $\mathrm{Mn}, \mathrm{Cr}, \mathrm{Zn}$ e $\mathrm{Pb}$, no compartimento do entorno da baía.

Essa distribuição evidencia que principalmente os elementos $\mathrm{Mn}, \mathrm{Pb}$ e Ba têm afinidade com as frações consideradas potencialmente disponíveis nos sedimentos de manguezal da região das ilhas da Baía de Camamu, enquanto que na região do entorno, foram os elementos $\mathrm{Mn}, \mathrm{Zn}, \mathrm{Cr}$ e Ba que mostraram essa associação mais estreita com as fases potencialmente disponíveis para o ambiente.

O efeito das atividades de mineração na baía de Camamu, em especial nas estações que compõem a região das ilhas, onde estão concentrados os principais setores do extrativismo mineiro na localidade, deve refletir o expressivo teor de metais, a exemplo do $\mathrm{Pb}$,
$\mathrm{Zn}, \mathrm{Mn}$ e $\mathrm{Ba}$, relacionado a todas as frações geoquímicas analisadas. Apesar do grande destaque desses elementos associados à fração residual (a exceção do $\mathrm{Ba}$ ), neste compartimento, suas associações à fração trocável (prontamente disponível para ser absorvida pela biota), se configuram como uma situação merecedora de maiores estudos e cuidados.

Lacerda (1994), estudando sedimentos de manguezais na Baía de Sepetiba, RJ, constatou que a fração oxidável predomina em mais de $60 \%$ para os vários metais considerados, principalmente $\mathrm{Zn}, \mathrm{Mn}$ e $\mathrm{Cu}$, enquanto que a fração redutível teve importância negligenciável para a maioria desses metais. Ao mesmo tempo, a fração trocável, imediatamente disponível para absorção pela planta foi considerada desprezível para a maioria dos metais, com exceção do $\mathrm{Mn}$, que correspondeu a $15 \%$ da concentração total, no estudo realizado. Resultados semelhantes foram encontrados por Salomons \& Förstner (1984), no estuário do rio Zaire, os quais relatam igualmente a importância dos substratos oxidáveis em sedimentos de manguezal em relação a sedimentos fluviais. Chiu \& Chou (1991) in Lacerda (1994), também relatam um aumento da

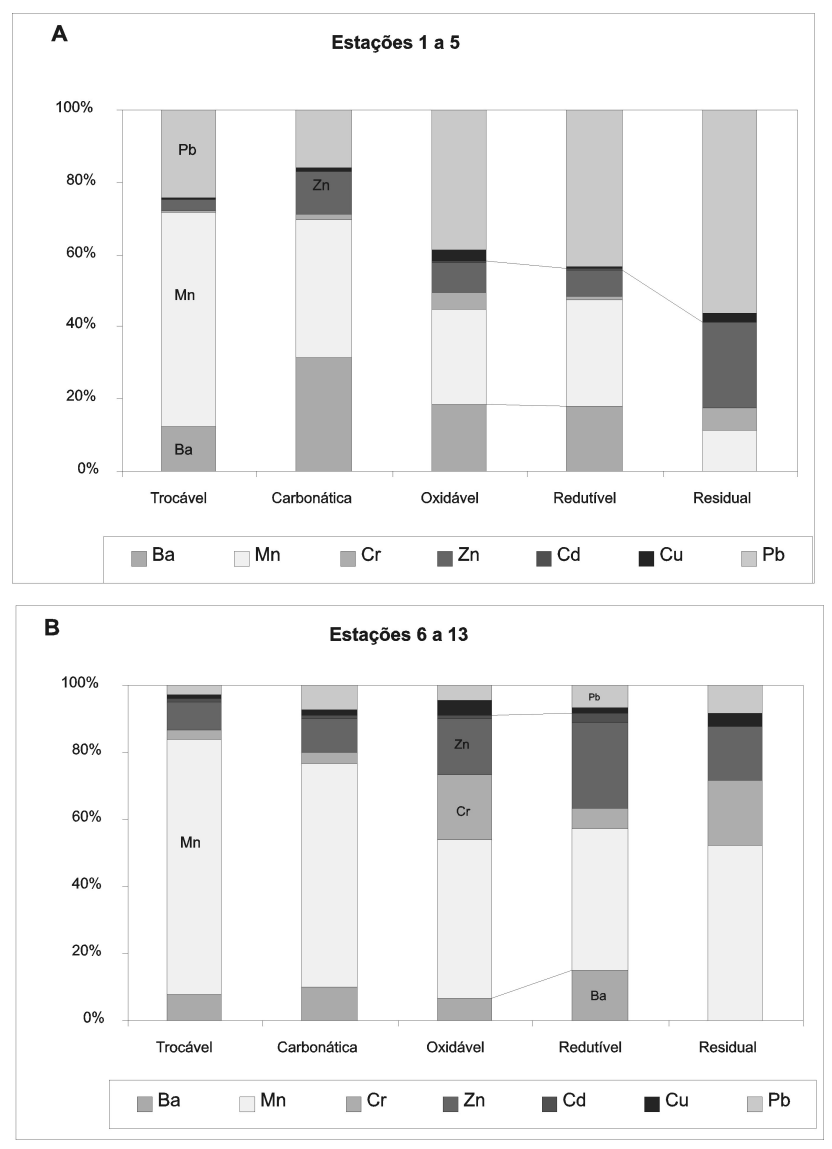

Figura 3 - Distribuição média de metais nas fases geoquímicas analisadas para cada fatia dos testemunhos amostrados nos sedimentos de manguezal da baía de Camamu. 
importância da fase oxidável para a distribuição de metais em sedimentos de manguezal em comparação com solos de floresta de bambu no rio Tamshi, Taiwan. Esses resultados evidenciam a importância dos sulfetos provenientes da decomposição anaeróbica da matéria orgânica na acumulação de metais pesados no ecossistema manguezal.

A Tabela 1 apresenta a eficiência analítica para as extrações seqüenciais, a qual foi testada realizando análises de extração total nas mesmas amostras utilizadas anteriormente para extrair as 05 (cinco) frações geoquímicas consideradas. Essa eficiência foi examinada através da relação entre os valores obtidos do somatório das diferentes fases geoquímicas e aqueles da extração total, para os diferentes elementos considerados nessas duas etapas analíticas. Foram considerados satisfatórios, os resultados que apresentaram variações inferiores a 10\%. Essa variação, entretanto, não foi considerada aceitável com relação ao Zn, Cu, Cd, Mn e Al. Esse fato pode ser atribuído à maior heterogeneidade das características intrínsecas dos elementos constituintes dos substratos dos dois compartimentos da Baía de Camamu. Fernandes (1991) constatou comportamento semelhante para $\circ \mathrm{Pb}$, estudando sedimentos fluviais e lagunares do RJ. Devese ressaltar ainda que alguns autores (Rapin et al., 1986; Campbell \& Tessier, 1984), têm relatado que a seletividade da técnica da extração seqüencial decai acentuadamente para sedimentos ricos em sulfetos (anóxicos), o que pode ter também contribuído para a dificuldade na obtenção de resultados satisfatórios, uma vez que regiões de manguezais se configuram como caracteristicamente anóxicas.

O grau de enriquecimento em metais é dependente do tamanho da partícula do sedimento e do conteúdo orgânico. As partículas de granulometrias finas, do tamanho silte ou argila e sedimentos ricos em material orgânico, tendem a favorecer uma maior adsorção de metais (Bubb \& Lester, 1995). Em sedimentos de manguezais, três grandes aspectos estão envolvidos na acumulação e distribuição de metais traços (Lacerda, 1998): a) acumulação preferencial por

Tabela 1 - Concentração média dos metais aplicando o método da extração seqüencial e da extração total.

\begin{tabular}{|c|c|c|c|c|c|c|c|c|}
\hline & $\begin{array}{c}\mathrm{Pb} \\
(\mu \mathrm{g} / \mathrm{g})\end{array}$ & $\begin{array}{c}\mathrm{Zn} \\
(\mu \mathrm{g} / \mathrm{g})\end{array}$ & $\begin{array}{c}\mathrm{Cr} \\
(\mu \mathrm{g} / \mathrm{g})\end{array}$ & $\begin{array}{c}\mathrm{Cu} \\
(\mu \mathrm{g} / \mathrm{g})\end{array}$ & $\begin{array}{c}C d \\
(\mu g / g)\end{array}$ & $\begin{array}{c}\mathrm{Mn} \\
(\mu \mathrm{g} / \mathrm{g})\end{array}$ & $\begin{array}{l}\mathrm{Fe} \\
(\%)\end{array}$ & $\begin{array}{c}\mathrm{Al} \\
(\%)\end{array}$ \\
\hline \multicolumn{9}{|l|}{ Etapas da extração seqüencial: } \\
\hline \multicolumn{9}{|l|}{ Região das ilhas (Estações 1 a 5) } \\
\hline Trocável & 10,59 & 1,10 & 0,17 & 0,20 & 0,11 & 28,97 & 0,01 & 0,01 \\
\hline L.D. & 0,7 & 0,27 & 0,09 & 0,24 & 0,21 & 0,8 & 0,01 & 0,01 \\
\hline Carbonática & 5,13 & 4,55 & 0,42 & 0,18 & 0,11 & 16,04 & 0,01 & 0,01 \\
\hline L.D. & 1,8 & 0,85 & 0,27 & 0,22 & 0,22 & 0,2 & 0,01 & 0,01 \\
\hline Oxidável & 111,30 & 25,22 & 9,53 & 8,09 & 0,73 & 78,13 & 0,86 & 0,27 \\
\hline L.D. & 3,0 & 1,7 & 3,7 & 1,7 & 1,31 & 2,1 & 0,02 & 0,09 \\
\hline Redutível & 131,01 & 22,84 & 2,64 & 0,59 & 1,00 & 90,07 & 0,14 & 0,05 \\
\hline L.D. & 2,7 & 3,57 & 1,7 & 0,28 & 2,0 & 3,73 & 0,04 & 0,01 \\
\hline Residual & 314,82 & 130,86 & 33,54 & 13,69 & 0,31 & 64,48 & 1,83 & 2,55 \\
\hline L.D. & 3,3 & 3,15 & 2,0 & 2,0 & 0,3 & 2,2 & 0,4 & 0,4 \\
\hline Total $(\Sigma)$ & 572,85 & 184,57 & 46,31 & 22,75 & 2,25 & 277,68 & 2,84 & 2,87 \\
\hline \multicolumn{9}{|c|}{ Região do entorno da Baía (Estações 6 a 13) } \\
\hline Trocável & 0,39 & 1,28 & 0,39 & 0,14 & 0,11 & 12,73 & 0,02 & 0,02 \\
\hline L.D. & 0,7 & 0,27 & 0,09 & 0,24 & 0,21 & 0,8 & 0,01 & 0,01 \\
\hline Carbonática & 0,90 & 0,86 & 0,31 & 0,15 & 0,11 & 8,24 & 0,01 & 0,01 \\
\hline L.D. & 1,8 & 0,85 & 0,27 & 0,22 & 0,22 & 0,2 & 0,01 & 0,01 \\
\hline Oxidável & 3,95 & 20,03 & 24,31 & 5,48 & 0,79 & 57,73 & 1,34 & 0,28 \\
\hline L.D. & 3,0 & 1,7 & 3,7 & 1,7 & 1,31 & 2,1 & 0,02 & 0,09 \\
\hline Redutível & 2,03 & 9,98 & 2,20 & 0,63 & 1,00 & 15,62 & 0,19 & 0,04 \\
\hline L.D. & 2,7 & 3,57 & 1,7 & 0,28 & 2,0 & 3,73 & 0,04 & 0,01 \\
\hline Residual & 12,70 & 25,20 & 30,97 & 5,82 & 0,27 & 82,27 & 0,93 & 1,88 \\
\hline L.D. & 3,3 & 3,15 & 2,0 & 2,0 & 0,3 & 2,2 & 0,4 & 0,4 \\
\hline Total $\left(\sum\right)$ & 19,96 & 57,34 & 58,18 & 12,23 & 2,29 & 176,59 & 2,49 & 2,22 \\
\hline \multicolumn{9}{|l|}{ Extração total: } \\
\hline Região das ilhas (Estações 1 a 5) & 536,56 & 176,92 & 43,99 & 19,54 & 0,06 & 194,29 & 2,72 & 4,84 \\
\hline Região do entorno da Baía (Estações 6 a 13) & 20,69 & 49,02 & 54,17 & 12,68 & 0,12 & 190,69 & 2,56 & 4,14 \\
\hline \multicolumn{9}{|l|}{$\begin{array}{l}\text { Relação entre o } \sum \text { das etapas da extração } \\
\text { seqüencial e da extração total: }\end{array}$} \\
\hline Região das ilhas (Estações 1 a 5) & 6,76 & 4,33 & 5,27 & 16,40 & 3962,59 & 42,92 & 4,36 & 40,66 \\
\hline Região do entorno da Baía (Estações 6 a 13) & 3,54 & 16,98 & 7,42 & 3,58 & 1805,15 & 7,39 & 2,55 & 46,32 \\
\hline
\end{tabular}


sedimentos de granulometria fina; b) acumulação de alto conteúdo de matéria orgânica e c) precipitação de sulfetos metálicos presentes como produto da sulfatoredução.

Os métodos de extração parcial são aplicados aos sedimentos para a obtenção de uma fração fracamente ligada a este material e são mais simplificados que os métodos de extração seletiva. Apesar deste método quantificar os elementos metálicos nos sedimentos, ele não permite avaliar quantitativamente a contribuição antropogênica e o risco potencial de liberação desses metais (Teixeira et al., 1997). Quando os metais são dissociados da matéria orgânica oxidada em suspensão, eles são eventualmente fixados em substratos reduzidos em sedimentos de manguezal, dessa forma eles terão a sua partição geoquímica alterada. Daí se fazer necessário a aplicação da extração seqüencial, para determinar os metais com diferentes associações geoquímicas no sedimento. $O$ ataque total foi utilizado com o objetivo de determinar a concentração total dos metais nos sedimentos, bem como avaliar a eficiência da extração seqüencial, através da comparação entre o somatório de todos os teores das frações geoquímicas estudadas com os teores obtidos da extração total.

A Tabela 2 apresenta os teores médios dos metais analisados pelo método de extração parcial. Verificase que não apresentam uniformidade de comportamento entre os dados analisados nas ilhas e da região do entorno da Baía. Os teores médios de $\mathrm{Pb}, \mathrm{Zn}$ e Cu foram mais elevados no primeiro compartimento, enquanto que no segundo compartimento o teores de $\mathrm{Mn}, \mathrm{Cr}, \mathrm{Al}$ e Fe apresentaram teores médios mais elevados.

Queiroz (1992), estudando o comportamento de alguns metais nos manguezais da Baía de Todos os
Santos, verificou que muitos dos metais considerados, inclusive alguns deles indicados como bastante tóxicos e encontrados sob forma relativamente acessível na natureza, como o $\mathrm{Pb}, \mathrm{Zn}, \mathrm{Cr}, \mathrm{Cu}, \mathrm{Cd}$, poderiam estar associados a uma série de atividades industriais, a exemplo da mineração. Queiroz (1992), constatou ainda que muitos desses cátions, apesar de não serem os elementos diretamente implicados nessas ações, podem participar das diferentes etapas intermediárias dos processos industriais, com conseqüente liberação para o ambiente durante essas atividades.

Comparações de dados deste estudo com outras áreas de manguezal do Estado da Bahia (Queiroz, 1992; Paredes et al., 1995, Santos 1997, Araújo 2000), mostrados na Tabela 2, permitiram constatar que o $\mathrm{Pb}$ apresentou, na região das ilhas da Baía de Camamu, os mais elevados teores relativos. Neste mesmo compartimento, o teor médio de Zn é comparável àquele da região de Santo Amaro, caracterizada por forte influência antropogênica (Queiroz, 1992; Paredes et al., 1995). Os teores dos demais elementos, bem como de todos os metais da região do entorno da Baía de Camamu, apresentaram-se comparáveis àqueles da região de Maragojipe, Joanes ou Alcobaça, consideradas ainda sob incipiente influência antropogênica.

Maiores investigações a respeito das associações geoquímicas desses metais nos sedimentos do manguezal da Baía de Camamu puderam ser realizadas através de métodos de extração seletiva.

\section{CONCLUSÕES}

A análise da concentração dos metais nos sedimentos, até a profundidade de $1 \mathrm{~m}$, revelou 0 comportamentos geoquímicos diferenciados para cada

Tabela 2 - Comparação entre as concentrações médias dos elementos Pb, Zn, Cr, Cu, Cd, Mn, Al e Fe, nos sedimentos do manguezal da Baía de Camamu com a de outras zonas de manguezal estudadas.

\begin{tabular}{|c|c|c|c|c|c|c|c|c|}
\hline Local & $\begin{array}{c}\mathrm{Pb} \\
\mu \mathrm{g} / \mathrm{g}\end{array}$ & $\begin{array}{c}\mathrm{Zn} \\
\mu \mathrm{g} / \mathrm{g}\end{array}$ & $\begin{array}{c}\mathrm{Cr} \\
\mu \mathrm{g} / \mathrm{g}\end{array}$ & $\begin{array}{r}\mathrm{Cu} \\
\mu \mathrm{g} / \mathrm{g}\end{array}$ & $\begin{array}{r}\mathrm{Cd} \\
\mu \mathrm{g} / \mathrm{g}\end{array}$ & $\begin{array}{l}\mathrm{Mn} \\
\mu \mathrm{g} / \mathrm{g}\end{array}$ & $\begin{array}{l}\mathrm{Al} \\
\% \\
\end{array}$ & $\begin{array}{l}\mathrm{Fe} \\
\% \\
\end{array}$ \\
\hline $\begin{array}{c}\text { Baía de Camamu } \\
\text { Região da llhas }\end{array}$ & 156,0 & 81,0 & 29,2 & 20,2 & $<1,0$ & 114,4 & 0,6 & 1,7 \\
\hline $\begin{array}{l}\text { Baía de Camamu } \\
\text { Região do entorno }\end{array}$ & 28,0 & 45,8 & 44,1 & 16,5 & $<1,0$ & 210,0 & 1,4 & 3,2 \\
\hline Maragojipe * & 9,6 & 59,9 & 73,2 & 15,0 & 0,06 & - & - & - \\
\hline Santo Amaro * & 95 & 84,9 & 142,1 & 56,8 & 9,4 & - & - & - \\
\hline Joanes ** & 18,72 & 23,57 & 15,73 & 11,8 & 0,59 & - & - & - \\
\hline Alcobaça *** & 29,16 & 5,43 & 69,2 & 8,73 & - & 700 & 1,9 & 2,9 \\
\hline
\end{tabular}


uma das zonas de manguezal individualizada (compartimento das ilhas e compartimento do entorno da Baía de Camamu).

Através da extração parcial, foi verificado que os teores de $\mathrm{Pb}, \mathrm{Zn}$ e $\mathrm{Cu}$ foram mais elevados no compartimento das ilhas, enquanto que o $\mathrm{Mn}, \mathrm{Cr}$, o $\mathrm{Al}$ e o Fe, apresentaram-se mais elevados no compartimento do entorno.

A dinâmica de partição dos metais nas diferentes fases geoquímicas pesquisadas por análises seqüenciais, a saber: trocável, carbonática, oxidável, redutível e residual, revelou que o efeito das atividades de mineração local é refletido no expressivo teor dos metais $\mathrm{Pb}, \mathrm{Zn}, \mathrm{Mn}$ e $\mathrm{Ba}$, relacionado a todas essas fases. A associação desses elementos à fase trocável no compartimento das ilhas, confirma a relação existente entre a atividade mineira/mineralização e o incremento dessas concentrações, configurando-se como uma situação merecedora de maiores cuidados.

A extração total revelou resultados satisfatórios com relação à extração seqüencial para $\mathrm{Pb}, \mathrm{Cr}$ e $\mathrm{Fe}, \mathrm{o}$ que pode ser atribuído a heterogeneidades nos constituintes dos substratos nos dois compartimentos da Baía de Camamu.

\section{REFERÊNCIAS}

Araújo, B.R.N. 2000. Diagnóstico Geoambiental de Zonas de Manguezal do Estuário do rio Itanhém Município de Alcobaça - Região Extremo Sul do Estado da Bahia. Dissertação de Mestrado. Instituto de Geociências, Universidade Federal da Bahia, UFBA, 159p.

Bradl, H.B. 2004. Adsorption of heavy metal ions on soils and soil constituents. Journal of Colloid and Interface Science, San Diego, 277(1)1-18.

Bubb J.M. \& Lester J.N. 1990. The impact of heavy metals on Lowland Rivers and the implications for man and environment. Sci. Tot. Environ., 100: 207233.

Cabral, A.R. \& Lefebvre, G. 1998. Use of selective sequential extraction technique as a tool in the study of heavy metal retention and release by soils with low clay contents. Water, Air and Soil Pollution, 102: 329-344.

Campbell, P.G.C. \& Tessier, A. 1984. Paleolimnological approaches to the study of acid deposition: metal partitioning in lacustrine sediments. In: Proc. Of Us Mine Vent. Symp., Epa Workshop On Paleolimnological Studies Of The History And Effects Of Acidic Precipitation. Rockport, Maine, $p$. 234-274.

Dolezal, J.; Povondra, P. \& Sulcek, Z. 1968. Decomposition Techniques in Inorganic Analysis:
Iliffe Books, London. with ammonium hydrogen sulphate. Analyst, 92, 239.

Fizman, M.; Pfeiffer, W. C. \& Lacerda, L. D. 1984. Comparasion of methods used for extraction and geochemical distribution of heavy metals in bottom sediments from Sepetiba Bay, R.J. Science and Technology Letters, 5:567-575.

Förstner, U. \& Wittmann, G. 1981. Metal Pollution in the Aquatic Environment. $2^{\mathrm{a}}$ ed. Berlin, Heidelberg, New York. Springer-Verlag, 486p.

Lacerda, L.D. 1998. Trace Metals Biogeochemistry and Diffuse Pollution in Mangrove Ecosystems. ISME. Mangrove Ecosystems Occasional Papers, $n \div$ 2, $65 p$.

Lacerda, L.D. 1994. Biogeoquímica de Metais Pesados em Ecossistemas de Manguezal. Tese para Prof. Titular. UFF, Niterói, RJ, 72p.

Lee, P.K; Baillif, P. \&Touray, J.C. 1997. Geochemical Behaviour and Relative Mobility of Metals (Mn, Cd, $\mathrm{Zn}$ and $\mathrm{Pb}$ ) in Recent Sediments of a Retention Pond Along the A-71 Motoway in Sologne, France. Environmental Geology, 32(2): 142-152.

Lim, P-E. \& M-Y Kiu, 1995. Determination and speciation of heavy metals in sediments of the Juru River, Penang, Malaysia. Envir. Monit. Assess. 35: 85-95.

Oliveira, O.M.C. de, Damasceno, R.N.; Queiroz, A. F. de S. \& Fahel Filho, E. 1998. Caracterização Geoambiental de Zonas de Manguezais da Baía de Camamu - BA: Subsídios para um Estudo Ambiental Sistemático. Revista Escola de Minas (REM), ano 62, vol. 51, nํ 4, pg. 42-46.

Oliveira, O.M.C. de. 2000. Diagnóstico Geoambiental em zonas de manguezal da Baía de Camamu BA. Tese de Doutorado em Geoquímica Ambiental. Universidade Federal Fluminense, RJ. 249p.

Paredes, J.F. Queiroz, A.F. de S.; Carvalho, I.G; Ramos, M.A.S.B.; Santos, A.L.F. \& Mosser, C. 1995. Heavy Metals in sediments: Mangrove swamps of the Subaé and Paraguaçu tributary rivers of Todos os Santos Bay, Bahia, Brazil. Série Tecnologia Ambiental, ํㅜ 9, Centro de Tecnologia Mineral, RJ, $15 p$.

Queiroz, A.F. de S. 1992. Mangroves de la baia de Todos os Santos - Salvador - Bahia - Brésil: ses caractéristiques et l'influence anthropique sur as géochimie. Tese de Doutorado. Université Louis Pasteur de Strasbourg, França. 148p.

Rapin, R. ; Tessier, A.; Campbeli, P.G.C. \& Carignan, R. 1986. Potential artifacts in the determination of metal partitioning in sediments by sequential extraction procedure. Environ.Sci. Technol. 20:836840.

Santos, A.L.F. 1997. Manguezais do Estuário do Rio Joanes - Lauro de Freitas - Bahia - Estudos de 
Caracterização Geoquímica como Subsídios para um Gerenciamento Ambiental Adequado. Dissertação de Mestrado em Geoquímica e Meio Ambiente. Instituto de Geociências, UFBA, 226p.

Salomons, W. \& Förstner, V. (1984). Metals in the Hidrocycle Berlin Springer-Verlag, 340pp.

Teixeira, E.C.; Sanchez, J.D.; Fernandes, I.D.; Formoso, M.L.L.; Pegorini, J. \& Pestana, M.H.D. 1997. Estudo preliminar dos metais em sedimentos de áreas de influência do processamento de carvão e da siderurgia - região do baixo Jacuí, RS. Geochim. Brasil. 11(1):039-052.

Tessier, A.; Campbell, P.G.C. \& Bisson, M. 1979. Sequential extraction procedure for the speciation of particulate trace metals. Analytica Chimica Acta, 51:844-850.

Yong, R.N.; Mohamed, A.M.O.; Warkentin, B.P. 1992. Principles of contaminant transport in soils. Amsterdam. Elsevier. $327 \mathrm{p}$.

Yong, R.N.; Galvez-Cloutier, R. \& Phadungchewit, Y. 1993. Can. Geot. J. 30:834-847. 